\title{
Elaboração de um instrumento de coleta de dados para identificação dos diagnósticos de enfermagem em parturiente*
}

\author{
The development of an instrument to collect data to \\ formulate nursing diagnoses for pregnant women
}

\author{
Elaboración de un instrumento de colecta de datos \\ para identificar los diagnósticos de enfermería de la parturiente
}

Satie Lúcia Nishimaru Sumita ${ }^{1}$, Ana Cristina de Freitas Vilhena Abrão², Heimar de Fátima Marin ${ }^{3}$

\section{RESUMO}

Objetivo: Construção de um instrumento de coleta de dados da parturiente. Métodos: as variáveis selecionadas foram os dados sociodemográficos, clínico-obstétricos e diagnósticos de enfermagem. Resultados: O instrumento foi estruturado e organizado com base na Taxonomia II - NANDA. Considerações finais: para facilitar o direcionamento da coleta de informações relevantes das pacientes e o manuseio das informações que levam ao julgamento clínico e que servirão como base na formulação do diagnóstico de enfermagem.

Descritores: Parto; Diagnóstico de enfermagem; Enfermagem obstétrica

\section{ABSTRACT}

Objective: The purpose of this descriptive study was to develop an instrument to collect dates for pregnant women. Methods: the variables was the demographic and clinical data to facilitate the determination of nursing diagnoses. Results: The structure and organization of the instrument was based on the NANDA-Taxonomy II Conclusions: to facilitate the best way to collect and manage important information that lead to adequate clinical judgment and formulation of nursing diagnoses.

Keywords: Parturition; Nursing diagnosis; Obstetrical nursing; Pregnant Women

\section{RESUMEN}

Objetivo: construcción de un instrumento de recolección de datos de la parturienta. Métodos: Las variables seleccionadas fueron los datos sociodemográficos, clínico-obstétricos y diagnósticos de enfermería. Resultados: El instrumento fue estructurado y organizado con base en la Taxonomía II - NANDA. Consideraciones finales: facilitar el direccionamiento de la recolección de informaciones relevantes de las pacientes y la manipulación de las informaciones que conducen al juicio clínico que, a su vez, servirán como base para la formulación del diagnóstico de enfermería.

Descriptores: Parto. Enfermería obstétrica. Diagnóstico de enfermería.

\footnotetext{
* Trabalho extraído da Tese de Doutorado apresentada na Universidade Federal de São Paulo - UNIFESP - São Paulo (SP), Brasil.

1 Professor Adjunto. Doutor em Enfermagem. Disciplina Enfermagem Obstétrica, Universidade Federal de São Paulo - UNIFESP São Paulo (SP), Brasil.

2 Professor Adjunto. Doutor em Enfermagem. Disciplina Enfermagem Obstétrica, Universidade Federal de São Paulo - UNIFESP São Paulo (SP), Brasil.

3 Professor Adjunto Livre Docente. Doutor em Ciências. Disciplina Enfermagem Obstétrica, Universidade Federal de São Paulo UNIFESP - São Paulo (SP), Brasil.
} 


\section{INTRODUÇÃO}

A busca de um modelo de assistência que melhor atenda às necessidades de cuidados das parturientes pautado nos princípios científicos, humanísticos, éticos e político-sociais é observada entre os profissionais comprometidos com a enfermagem obstétrica. O modelo biomédico constituiu a base para a prática da enfermagem durante muitos anos, mas nas últimas décadas, vem sendo questionado e substituído por outros que melhor retratem, atendam e complementem a estrutura da assistência às mulheres durante a parturição.

Observa-se, também, uma preocupação na prestação de cuidados de enfermagem baseada em evidências científicas e o uso do processo de enfermagem como metodologia para a prática e documentação em enfermagem.

O processo de enfermagem é um instrumento e método científico que direciona o trabalho dos enfermeiros, auxiliando na determinação das necessidades do cliente, na tomada de decisões, planejamento e implementação da assistência e avaliação das conseqüências do atendimento prestado ${ }^{(1-3)}$.

A primeira fase do processo de enfermagem, denominada de Histórico de enfermagem por Horta ${ }^{(4)}$; de avaliação inicial por Stanton, Paul e Reeves ${ }^{(1)}$ e de Assessment por Carpenito ${ }^{(5)}$, baseia-se na coleta sistemática de informações referentes ao cliente, ao sistema do cliente, da família ou comunidade.

Esta fase é considerada como a etapa primordial do processo, pois fornece informações, define as necessidades do paciente, permite à enfermeira identificar o diagnóstico de enfermagem e planejar o atendimento ${ }^{(6-7)}$. Constitui-se na etapa que fundamenta as demais fases do processo de enfermagem. Ao realizar o levantamento de dados é preciso se concentrar na identificação do estado de saúde, do padrão que o cliente/paciente usa para resolução dos problemas, no estado funcional, na resposta à terapêutica, no risco para problemas potenciais e no desejo de um nível mais alto de bem-estar ${ }^{(5)}$. Esta fase inicial consiste em coletar, organizar e documentar metodicamente os dados apreendidos, pois o processo de avaliação das necessidades é dinâmico e constante, da mesma forma que os outros componentes do processo de enfermagem.

Vale salientar, também, o exponencial aumento no volume de informações que precisam ser documentadas no prontuário do paciente.

Neste aspecto, a documentação em enfermagem atinge um ponto crítico, uma vez que pode representar a ferramenta para tornar a profissão mais visível, permitindo análises e avaliações das ações de enfermagem. Por outro lado, o que não está documentado, embora, possa ter sido realizado, em termos práticos não existe.
E, via de regra, nos prontuários de parturientes, a documentação dos dados relacionados à assistência de enfermagem prestada é escassa ${ }^{(8)}$.

$\mathrm{Na}$ área obstétrica, inclui-se um outro fator determinante na ação e documentação de enfermagem, a agilidade. A avaliação das condições de nascimento e assistência de enfermagem é um processo rápido, caracterizado por técnicas mais ágeis. Com freqüência, nos prontuários obstétricos, o que mais se identifica é a documentação dos dados epidemiológicos, como tipo de parto, valores de Apgar e o peso do recém-nascido, deixando de evidenciar a essência da assistência de enfermagem prestada desde a admissão até a alta neste setor.

Embora a prática do atendimento ao parto tenha se modificado com os progressos técnico-científicos que ocorreram após a introdução de técnicas cirúrgicas, anestesia, hemoterapia, antibioticoterapia, com os avanços notáveis na vigilância, diagnóstico, terapias fetais e com a introdução de terapias alternativas na assistência, busca-se ainda por maior qualidade de assistência durante o processo do nascimento.

Neste aspecto, alguns autores, inclusive ${ }^{(9)}$ citam que a mortalidade materna afeta mais aos indivíduos socioeconomicamente carentes, pois muitos desses óbitos ocorrem por falta de recursos adequados e por erros de tratamento pela equipe obstétrica como falta de acompanhamento cuidadoso à mulher e ao feto durante o trabalho de parto, parto e o início do puerpério.

Deve ser considerado, também, que o Centro Obstétrico, em geral, é um local que apresenta várias fontes de estresse às parturientes, com situações de urgência e algumas emergências, que caracterizam as múltiplas necessidades das parturientes. Tais fatores determinam que o instrumento de levantamento de dados seja coerente com as características do setor, respeitando a quantidade de informações solicitadas e o tempo gasto para sua aplicação.

Dadas as características de um centro obstétrico, a busca pela qualidade da assistência de enfermagem por meio da implementação do processo de enfermagem com caráter legal, ético e técnico, é um desafio a ser enfrentado e uma oportunidade para organizar o atendimento e viabilizar a análise de sua efetividade.

$\mathrm{Na}$ documentação do processo, é essencial o uso de uma terminologia que permita futuras análises e comparações. Diversas terminologias e sistemas de classificação em enfermagem foram desenvolvidos. Porém, a enfermagem não conta ainda com um único sistema que possa ser suficiente para representar o universo de ação da enfermagem. Estudos como este têm sido desenvolvidos para avaliar e nutrir tais sistemas de vocabulários. Como regra geral, vale destacar que a terminologia selecionada para documentação dos dados deve ser modeloindependente para que seja usada por enfermeiros do 
mundo todo, independente do modelo assistencial que a instituição tenha adotado para direcionar o cuidado de enfermagem ${ }^{(3)}$.

Pela aplicação do processo de enfermagem na assistência à parturiente, a enfermeira pode melhorar a assistência, obter maior segurança para identificar o estado de saúde e ou problemas, fazer o planejamento, implementar as intervenções e realizar a avaliação da assistência de enfermagem, melhorando a assistência e o ensino na área.

Em resposta a este desafio, e usufruindo a oportunidade, também pela falta de pesquisas e publicações nesta área em nosso meio, este estudo teve como objetivo elaborar um instrumento estruturado para a coleta de dados na assistência à parturiente, possibilitando a identificação de diagnósticos, base das intervenções de enfermagem.

\section{MÉTODOS}

Trata-se de um estudo descritivo sobre a construção de um instrumento de coleta de dados da parturiente. As variáveis selecionadas foram os dados sociodemográficos, clínico-obstétricos e os diagnósticos de enfermagem. Para a elaboração do instrumento de coleta de dados optou-se pela Taxonomia II da Associação Norte Americana de Diagnósticos de Enfermagem - NANDA ${ }^{(10)}$, como estrutura de organização para a coleta de dados e linguagem padronizada de registro clínico. Objetivando facilitar a aplicação do instrumento de coleta de dados na prática assistencial em Centro Obstétrico, decidiu-se elaborar perguntas abertas e fechadas, dando preferência às fechadas, do tipo "check-list", que servem como guia para que os dados considerados essenciais não sejam omitidos e prováveis erros de registro não sejam cometidos.

Ainda com a intenção de facilitar a análise, interpretação e agrupamento das informações coletadas, criouse um sistema com as variáveis de estudo contemplando os Domínios e suas Classes, os possíveis diagnósticos de enfermagem em conformidade com a definição, as respectivas características definidoras e os fatores relacionados ou de riscos.

Ao se estabelecerem as possibilidades diagnósticas, observou-se a necessidade de inserir alguns diagnósticos, características definidoras e fatores relacionados ou de riscos que não constam na classificação NANDA. Esta iniciativa foi encorajada em parte pela recomendação de se utilizar os diagnósticos ainda não aprovados, como forma de também incentivar o desenvolvimento ordenado e científico de novos diagnósticos de enfermagem para futuras inclusões ${ }^{(5)}$.

A etapa seguinte foi submeter o instrumento de coleta de dados da parturiente para ser avaliado por especialistas quanto às necessidades da parturiente e se os dados auxiliavam na identificação dos diagnósticos de enfermagem.

Após contato verbal e concordância em participar desta etapa do trabalho, foi enviado o formulário a três enfermeiras especialistas; todas com mais de dez anos de experiência na área de assistência de enfermagem à parturiente. Vale ressaltar que uma das enfermeiras também possuía experiência na aplicação da sistematização da assistência de enfermagem, identificação dos diagnósticos de enfermagem e intervenções. O tempo de retorno variou de três a cinco dias.

Com base nestas considerações, o instrumento de coleta de dados da parturiente foi elaborado, procurando viabilizar sua implementação no Centro Obstétrico, sabendo que o tempo médio de permanência da parturiente neste setor é cinco horas, em média.

\section{RESULTADOS}

O formulário de coleta de dados da parturiente constituiu-se de Dados de Identificação, dos Domínios e suas classes, com a introdução de uma coluna lateral contendo possíveis Diagnósticos de Enfermagem que podem ser selecionados pelo profissional. 


\section{FORMULÁRIO DE COLETA DE DADOS DA PARTURIENTE}

\section{A - IDENTIFICAÇÃO}

$\mathrm{RH}$ : |_L_L_L___ Nome: Idade:

Data: ____ _ Hora: ___ Tempo transcorrido da admissão até a coleta de dados:

\begin{tabular}{|c|c|}
\hline B-DOMÍNIOS: classes & $\begin{array}{l}\text { C- DIAGNÓSTICO DE } \\
\text { ENFERMAGEM }\end{array}$ \\
\hline $\begin{array}{l}\text { RELACIONAMENTOS I PAPEL: Relacionamento familiar } \\
\text { Idade: } \\
\text { Situação Conjugal: } \\
\text { A gravidez foi planejada: } \square \text { Sim } \square \text { Não; no momento ela é desejada: } \square \text { Sim } \square \text { Não } \\
\text { Outros dados: }\end{array}$ & $\begin{array}{l}\text { Risco } \mathrm{p} / \text { maternidade } \\
\text { prejudicada } \\
\square \text { Outro }\end{array}$ \\
\hline 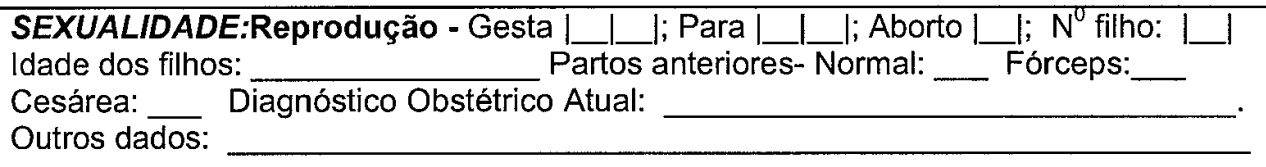 & $\begin{array}{l}\square \text { Risco p/lesão } \\
\text { materna-fetal } \\
\square \text { Outro }\end{array}$ \\
\hline $\begin{array}{l}\text { PROMOÇÃO DA SAÚDE: Gerenciamento à saúde } \\
\text { Fez Pré-natal- } \square \text { Não } \square \text { Sim } \quad N^{0} \text { Consultas: } \\
\text { Teve orientação sobre trabalho de parto/parto } \square \text { Não } \square \text { Sim; } \\
\text { Outros dados: }\end{array}$ & $\begin{array}{l}\square \text { Comportamento de } \\
\text { busca de saúde } \\
\square \text { Manutenção da saúde } \\
\text { ineficaz } \\
\square \text { Outro: }\end{array}$ \\
\hline 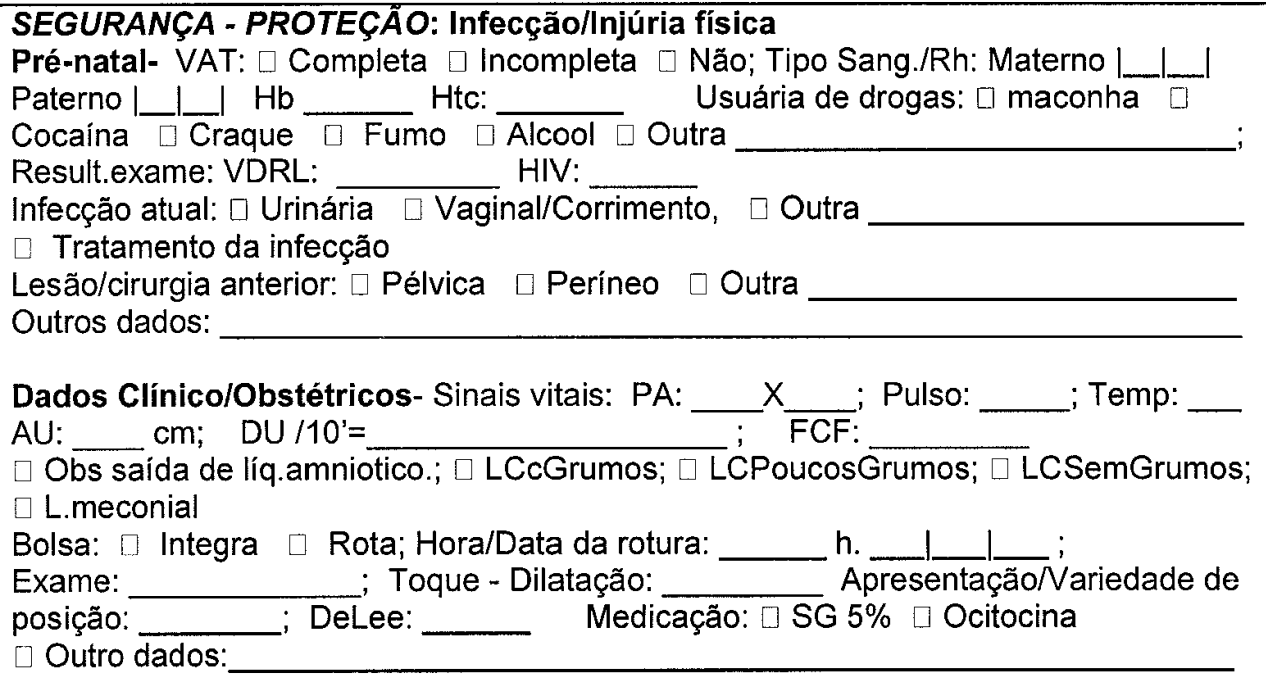 & $\begin{array}{l}\square \text { Proteção ineficaz } \\
\text { (materno/fetal) } \\
\square \text { Risco p/ lesão } \\
\square \text { Risco p/ infecção } \\
\square \text { Integridade da pele } \\
\text { prejudicada } \\
\square \text { Risco / } \square \text { Perfusão } \\
\text { útero placentária } \\
\text { ineficaz } \\
\square \text { Outro }\end{array}$ \\
\hline 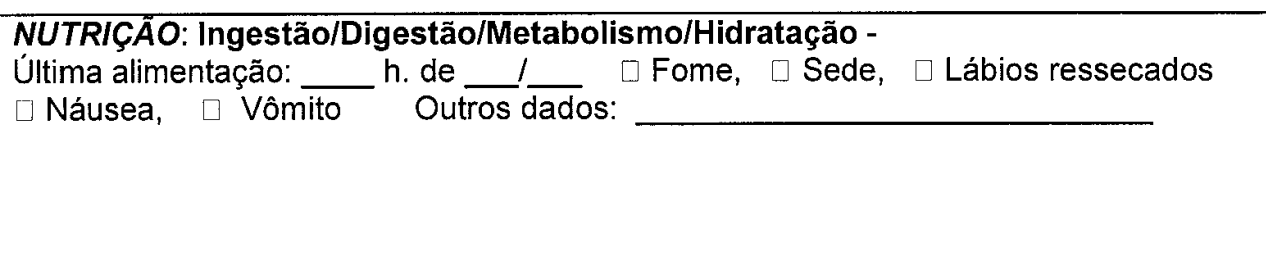 & $\begin{array}{l}\square \text { Nutrição desequili- } \\
\text { brada } \\
\square \text { Náusea } \\
\square \text { Risco p/ volume de } \\
\text { líquido desequilibrado } \\
\square \text { Outro } \\
\end{array}$ \\
\hline 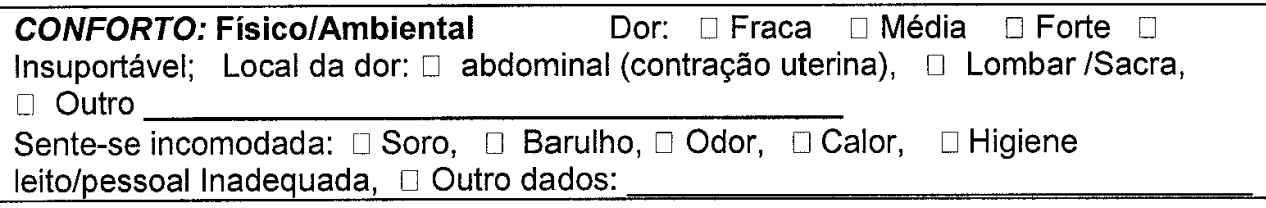 & $\begin{array}{l}\text { Dor aguda } \\
\square \text { Conforto prejudicado } \\
\square \text { Outro } \\
\end{array}$ \\
\hline
\end{tabular}


continuação...

\begin{tabular}{|c|}
\hline $\begin{array}{l}\text { ELIMINAÇÃO: Urinária//Vaginal/Gastrointestinal - } \\
\text { El. urinária: } \square \text { Polaciúria, } \square \text { Retenção urinária, } \square \text { Outra } \\
\text { El. vaginal: } \square \text { Líquido amniótico, } \square \text { Sangramento, } \square \text { Outra } \\
\text { El. intestinal: } \square \text { Normal, } \square \text { Diarréia, } \square \text { Constipação; } \\
\text { Evacuou após última refeição: } \square \text { Sim } \square \text { Não }\end{array}$ \\
\hline $\begin{array}{l}\text { ATIVIDADE/REPOUSO: Sono-Repouso/ Balanço energético/ Atividade-exercício } \\
\text { IResposta pulmonar/Cardiovascular- } \\
\mathrm{N}^{0} \text { noite sem dormir: I_l; } \square \text { Cansaço; } \square \text { Fadiga; } \square \text { Respiração p/ boca; } \\
\square \text { Outra } \\
\text { Faz exercício de respiração/relaxamento/alívio da dor: } \square \text { Sim, } \square \text { só cl orientação, } \\
\square \text { Não deambula: } \square \text { Sim, } \square \text { Com ajuda, } \square \text { Não } \square \text { Edema, local: } \\
\square \text { Varizes, local } \\
\square \text { Hemorróida; } \square \text { Outros dados: }\end{array}$ \\
\hline
\end{tabular}
; $\square$ Hemorróida; $\square$ Outros dados:

Eliminação urinária prejudicada

$\square$ Eliminação vaginal comprometida

$\square$ Eliminação intestinal ineficaz

$\square$ Outro:

$\square$ Distúrbio do padrão

do sono

$\square$ Fadiga

$\square$ Padrão resp.ineficaz

Mobilidade física

prejudicada

$\square$ Perfusão periférica ineficaz

$\square$ Outro:

\section{PERCEPÇÃO/COGNIÇÃO: Atenção/Orientação/Sensação-} percepção/Cognição/Comunicação -

Gostaria de ter esclarecimento sobre algum assunto: $\square$ Não $\square$ Sim; se sim, que assunto: $\square$ Trabalho de parto; $\square$ Exames realizados; $\square$ Condições do feto/bebê; \lfloor] Tipos de parto; $\square$ Tipos de anestesia; $\square$ Técnicas p/alívio da dor;

Outros assuntos

Compreensão: $\square$ Boa, $\square$ Moderada, $\square$ Comprometida. Outros dados:

\section{ENFRENTAMENTO/TOLERÂNCIA AO ESTRESSE: resp. póstrauma/} neurocomportamentais

Há algo que a amedronta: $\square$ Não, $\square$ Sim , se sim, o que ? $\square$ Dor, $\square$ Parto, $\square$ Ficar só no quarto, $\square$ Morrer, $\square$ Outro

$\square$ Agitação $\square$ Choro, $\square$ Grito, $\square$ Voz trêmula, $\square$ Manifesta preocupação

[] Outros dados:

\section{AUTOPERCEPÇÃO: autoconceito/auto estima/ imagem corporal}

$\square$ Relutância na aceitação da terapêutica; $\square$ Hesitante em tentar novas situações;

$\square$ Refere não ter energia/força; $\square$ Incapaz de lidar com os acontecimentos;

$\square$ Constrangimento durante o exame ginecológico; $\square$ Outra

$\square$ Outros dados:

PRINCIPIOS DE VIDA: coerência de valores/crenças/ação congruente Religião: $\square$ Não $\square$ Sim, Faz alguma limitação ao tratamento: $\square$ Não $\square$ Sim, qual:

$\square$ Outros dados:

Coren:

\section{Enfermeira}

A seguir, os itens e as justificativas que o compõem, são descritos.

\section{1 - Identificação}

Inicia-se com variáveis que permitem a identificação (Item A) e o estudo do tempo de internação em Centro Obstétrico, a saber: nome, data e horário da admissão e da coleta de dados.

Os dados de identificação são coletados para fins de arquivamento, estatístico e pericial, além de fornecerem subsídios para individualizar a assistência ao paciente ${ }^{(11)}$.
Saber o nome do paciente é fundamental para iniciar o processo de relacionamento e estabelecer a relação de confiança, no caso entre a parturiente e a enfermeira. Não se deve identificar uma pessoa pelo diagnóstico clínico ou obstétrico ou pelo número do leito. Este tipo de atitude, além de desrespeitosa, cria resistência, dificuldades e desconfiança no relacionamento profissional entre o prestador do cuidado e a cliente/paciente.

A data da admissão tem como finalidade a documentação, a identificação e a localização da parturiente no hospital. O horário da admissão permite conhecer o tem- 
po de internação hospitalar e de trabalho de parto, dado este de fundamental importância na assistência obstétrica, por permitir avaliar a evolução obstétrica. Com o evoluir do trabalho de parto, as necessidades da parturiente, por certo, sofrerão alterações, assim como novas intervenções serão necessárias.

\section{2 - Domínios, Classes e Diagnóstico de Enfermagem}

Os itens $\mathrm{B}$ e C do formulário referem-se às variáveis que caracterizam os diagnósticos de enfermagem. Foram selecionados as características definidoras e os fatores relacionados ou de riscos, relacionando-os aos domínios e classes, segundo a Taxonomia II da NANDA.

- Relacionamento / Papel: este domínio contém três classes das quais apenas a classe Papéis do cuidador foi selecionada. Coletar dados a respeito da idade, situação conjugal, fonte de rendimento, se a gravidez foi planejada e se é ou não desejada, possibilita à enfermeira fazer a correlação com outros dados como a idade e a paridade, avaliar a relação familiar, identificar as respostas individuais para a nova condição de papéis, especialmente no momento do trabalho de parto, avaliando a participação no autocuidado e auxiliando no planejamento geral da assistência de enfermagem.

Dentre os possíveis diagnósticos, relacionou-se o "Risco para maternidade prejudicada". Embora a gestação seja geralmente considerada um período de grande alegria, fatores como a falta de planejamento associados à gravidez não desejada, à imaturidade psíquica, emocional e social para assumir novas responsabilidades, bem como a falta de apoio familiar, podem fazer com que este período seja de muita angústia, sofrimento e insatisfação, aumentando o risco para que a maternidade transcorra de forma alterada.

Segundo Carpenito ${ }^{(5)}$, indivíduos ou famílias que podem ter alto risco para desenvolvimento ou experiência de dificuldades de paternidade/maternidade são os relacionados a pais solteiros, emocionalmente perturbados, adolescentes, alcoólatras, viciados em drogas, deficientes agudos, vítimas de acidentes, os relacionados aos filhos de uma gestação indesejada, deficiente físico, de um sexo indesejado ou com características indesejadas.

- Sexualidade: das três Classes que possui, considerou-se apenas a Classe Reprodução e foi introduzido o diagnóstico "Risco para lesão materno-fetal". Esta variável é identificada pelo número de gestações (gesta), número de paridade (para), número de abortos, número de filhos, número e via de parto anterior e diagnóstico obstétrico. A multiparidade, abortamentos e partos sucessivos, mesmo fisiológicos, condicionam na fibra muscular uterina, modificações que a tornam predisposta à ruptura uterina ${ }^{(12)}$. Assim, o risco é ainda maior nas parturientes multíparas e com cesáreas anteriores. Portan- to, os dados desta variável permitem à enfermeira relacionar os fatores que podem ou não aumentar riscos e caracterizar o diagnóstico de "Risco para lesão materno fetal" e, assim, podem contribuir para o planejamento de medidas que previnam ou minimizem os efeitos do risco obstétrico.

- Promoção da Saúde: a classe Controle da saúde foi selecionada. O conhecimento desta variável permite a enfermeira identificar diagnósticos como: "Comportamento de busca de saúde materno-fetal" e "Manutenção ineficaz da saúde". Existe promoção da saúde, quando se identificam comportamentos que demonstram desejo de buscar um nível mais elevado de bem-estar, durante ou fora do período gestacional e/ou evidência de maior controle sobre as práticas de saúde, como a freqüência ao pré-natal, realização ou seguimento das principais medidas recomendadas durante a gestação, participação em grupos de orientação, leituras sobre assuntos relacionados à saúde, questionamentos e participação no cuidado durante o trabalho de parto, ausência de comportamentos de risco tais como: tabagismo, drogas, álcool, obesidade e outros.

- Segurança-Proteção: das seis classes existentes neste domínio, foram selecionadas Infecção e Injúria física. As variáveis pré-natais, clínicas e obstétricas, que sustentam a elaboração diagnóstica são: hábitos, problemas de saúde pregressa e atual, esquema de vacina antitetânica, resultados laboratoriais, sinais vitais maternos, altura uterina, dinâmica uterina, freqüência cardíaca fetal, evolução do trabalho de parto (tempo de trabalho de parto e número de toques), infusão venosa, uso de ocitocina, presença ou não de infecção em decorrência da ruptura prematura ou precoce das membranas ovulares, mecônio ou fisometria. Outra variável considerada foi o corrimento vaginal, também conceituado como anormalidade do conteúdo vaginal, seja em quantidade ou em aspecto físico que se exterioriza por meio dos órgãos genitais externos ${ }^{(13)}$. A identificação desta característica é feita baseada na anamnese e na inspecção (secreção de cor amarelado/esverdeado, bolhoso, odor fétido e quantidade aumentada). Os fatores relacionados considerados foram a ruptura das membranas ovulares e processos infecciosos. A existência de lesão ou cirurgia prévia permite ao enfermeiro analisar e associar outros fatores relacionados ou de riscos, identificando os possíveis diagnósticos de enfermagem, tais como: "Risco para perfusão tissular cardiopulmonar ineficaz", "Risco para infecção", "Integridade da pele prejudicada", "Risco para lesão materno-fetal" e "Proteção materno-fetal ineficaz". Foi introduzido o diagnóstico "Risco para perfusão útero placentária ineficaz", e considerados como fatores de risco, o trabalho de parto, o uso de medicação (tais como ocitocina e meperidina) e a ruptura das membranas ovulares. 
No trabalho de parto, a repetição das contrações uterinas rítmicas cria uma situação na qual, durante a contração, existe a possibilidade da pressão intramiométrica ultrapassar a pressão intravascular sangüínea, interrompendo parcial ou completamente, o fluxo sangüíneo para a placenta e espaço interviloso; embora, normalmente, o feto possua reservas suficientes para tolerar essa interrupção intermitente das trocas de dióxido de carbono e oxigênio, quando há atividade uterina excessiva, redução da superfície placentária, redução da pressão sangüínea materna e aumento das demandas fetais, essa tolerância pode ser ultrapassada e a ausência de oxigênio suficiente para, através do ciclo de Krebs, suprir as necessidades energéticas do feto resulta na formação do ácido lático, que traz danos ao sistema nervoso, podendo levar à morte fetal ${ }^{(9)}$.

No que se refere à medicação, mais especificamente, ao uso de ocitocina, considerou-se a possibilidade da estimulação ser excessiva e provocar hiperatividade uterina e, conseqüentemente, a hipóxia fetal. Ao uso da meperidina, considerou-se o fato de que este opióide atravessa a placenta e age sobre o sistema nervoso central do feto, deprimindo-o. Os efeitos deste opióide no recém-nascido dependerão da dose, via de administração e intervalo entre a administração materna e o momento do nascimento ${ }^{(14)}$.

Quanto aos fatores relacionados à ruptura das membranas ovulares, considerou-se a possibilidade de compressão do cordão umbilical pela oligodramnia e o aumento das contrações uterinas. Conhecer estes fatores de riscos permite elaborar intervenções que possam atenuar ou prevenir o estabelecimento real do problema.

- Nutrição: as classes selecionadas para este estudo foram: ingestão e hidratação. Os dados referentes ao horário da última refeição, a presença de sede, fome e lábios ressecados permite relacioná-los com as condições clínicas e/ou obstétricas, identificando possíveis diagnósticos de "Nutrição desequilibrada", "Náusea" e "Risco para volume de líquido desequilibrado" com a finalidade de propor intervenções que possam atender às necessidades da parturiente.

- Conforto: na Classe físico e ambiental, foi introduzido o diagnóstico "Conforto prejudicado", e as características definidoras consideradas foram: dor, calor, sede, higiene inadequada (pessoal e/ou do leito), barulho e outras. Os desconfortos relatados pelas parturientes estão relacionados aos aspectos físicos e ambientais. $\mathrm{O}$ físico, em geral, é ocasionado na parturiente por contrações uterinas, compressão e estiramento dos ligamentos pélvicos e dorsais, provocando a dor. As informações referentes à dor, sua intensidade e localização, bem como o conhecimento dos fatores que levam a parturiente a sentir-se incomodada, permite a avaliação das possibilidades diagnósticas da $d o r$ e conforto prejudicado pela enfermeira.
De acordo com a classificação NANDA, a dor do trabalho de parto é do tipo aguda. As mulheres sentem dor durante o trabalho de parto. Entretanto, as respostas de cada parturiente são diferentes, devendo ser analisadas individualmente, já que a dor é sempre uma experiência subjetiva e pessoal. Para avaliar a intensidade da dor, devem ser consideradas não só as características das contrações uterinas, mas as repercussões sociopsicocomportamentais envolvidas no processo da parturição. Embora seja difícil mensurar a intensidade dolorosa e o grau de reação a ela, pois a dor é uma sensação subjetiva e influenciável por vários fatores.

Alguns instrumentos foram construídos para aferir a intensidade da dor. Tais instrumentos diferem na aplicação, conforme a idade, compreensão, abstração e verbalização dos pacientes com dor ${ }^{(15)}$. Dentre as escalas desenvolvidas para mensurar a intensidade da dor, a mais usada entre os indivíduos adultos com compreensão e verbalização satisfatória, é o auto-relato.

Neste estudo, as escalas consideradas para avaliação da intensidade dolorosa em parturientes foram o autorelato com avaliação verbal aferida, de acordo com os seguintes parâmetros: sem dor, dor fraca ou leve, dor moderada, dor intensa e dor insuportável. A identificação das contrações uterinas quanto à freqüência, duração e intensidade e as evidências comportamentais observadas, tais como as manifestações de agitação, grito, choro, proteção da parte dolorida, sudorese, taquipnéia, taquicardia, náuseas, vômitos, debilidade, palidez e outros sinais e sintomas permitem sustentar a afirmação de presença de dor. Vale ressaltar que a dor é tanto um diagnóstico de enfermagem como uma característica definidora para o diagnóstico "conforto prejudicado", introduzido neste estudo. Os fatores relacionados considerados foram agentes físicos, biológicos e psicológicos do trabalho de parto.

Em desconforto ambiental, observa-se a condição de higiene do leito, os ruídos e a temperatura ambiental e a percepção da parturiente frente a estes fatores.

- Eliminação: as Classes selecionadas para este estudo foram: Sistema urinário e Sistema gastrointestinal. Quanto ao possível diagnóstico relacionado ao sistema urinário, considerou-se a "Eliminação urinária prejudicada". A diminuição mais acentuada do fluxo urinário é observada quando se está em decúbito dorsal em razão da compressão dos ureteres pelo volume uterino. Além disto a bexiga distendida pode obstruir a descida do feto ${ }^{(16)}$.

Na Classe Sistema Gastrintestinal, foi também acrescentado o diagnóstico "Eliminação intestinal ineficaz", caracterizado por eliminação ausente após a última refeição e presença de massa retal palpável pelo toque vaginal. Os fatores relacionados considerados foram o hábito irregular e a falta de estímulo/tempo hábil para efetuar a eliminação. 
A maior preocupação com relação a eliminação intestinal é evitar a contaminação fecal no parto e o constrangimento que esta pode causar, justificando assim, a importância de estimular a eliminação espontânea antes do parto.

Neste domínio, foi introduzida a Classe "Sistema Reprodutivo". Nesta, destaca-se o diagnóstico "Eliminação vaginal comprometida" caracterizado pela presença de sangramento relacionado às lesões útero placentárias ou de trajeto. Ainda, decidiu-se estabelecer que as características definidoras presença de líquido amniótico e secreções vaginais com características consideradas comprometedoras, estão relacionadas aos diagnósticos: perfusão placentária/ tissular prejudicada e risco para infecção.

- Atividade e Repouso: foram selecionadas as classes: Sono-Repouso, Atividade-exercício, Equilíbrio de energia, Respostas Cardiovasculares e pulmonares. No que se refere ao sono e repouso, as queixas por dificuldades para dormir são comuns ao final da gestação e são em geral ocasionadas pelo desconforto relacionado à falta de posição em razão do aumento no volume uterino, pela dificuldade para respirar, pela dor em especial na região lombar, além da ansiedade manifestada pela maioria das gestantes. Durante o trabalho de parto, o fenômeno compressivo aortocava na posição supina é intensificado pelas contrações uterinas, provocando menor fluxo sangüíneo e conseqüente comprometimento do estado fetal. Isto deve ser fator de atenção e observação no levantamento de dados, para estabelecer intervenção adequada.

Com relação a Respostas Cardiovasculares e pulmonares, vale lembrar que as contrações uterinas ocasionam aumento na pressão sangüínea materna durante o primeiro e o segundo período do parto, retornando ao nível anterior a ele no terceiro período. Outros fatores causadores de elevação da pressão sangüínea são a ansiedade, a apreensão e a dor. $\mathrm{O}$ aumento da freqüência cardíaca durante o segundo período é em razão dos esforços praticados no trabalho de parto e parto ${ }^{(17)}$. Durante a fase final do trabalho de parto pode ocorrer um aumento de $300 \%$ da ventilação, quando comparada ao estado não-gravídico. Nesta fase, dá-se a hiperventilação em resposta à dor, que é outro aspecto relacionado à classe atividade-exercício, na qual a posição supina pode resultar na redução de $20 \%$ da capacidade funcional residual $^{(18)}$. Esta postura é associada, possivelmente, a certo grau de obstrução do fluxo expiratório.

Em relação a Atividade-exercício, destaca-se que a deambulação durante a dilatação encurta a duração do parto $^{(19)}$. Ainda, as posições verticais (sentada e em pé) favorecem o funcionamento respiratório e metabólico, além de beneficiar o feto, favorecendo um melhor equilíbrio ácido-base na artéria umbilical ${ }^{(20)}$.

Embora, estudos evidenciem tais benefícios, observa- se, ainda, alguns fatores que interferem na realização destas práticas, tais como: o uso rotineiro de medicação endovenosa contínua com bomba de infusão e uso de analgesia (meperidina) dificultando a livre deambulação. Assim, tendo em vista tais fatores de atenção para o cuidado materno-fetal, as possibilidades diagnósticas incluídas nesta classe foram: "Distúrbio no padrão de sono", "Fadiga", "Padrão respiratório ineficaz", "Mobilidade física prejudicada" e "Perfusão placentária/tissular materno-fetal ineficaz".

- Percepção/Cognição: as classes selecionadas foram Atenção, Orientação, Cognição, Sensação/percepção e Comunicação. O conhecimento sobre escolaridade, oportunidade de obter informações no pré-natal, capacidade de compreender a informação e se a parturiente gostaria de receber esclarecimentos sobre algum assunto, permitem à enfermeira identificar os possíveis problemas ou diagnósticos de enfermagem de "Déficit de conhecimento" sobre os procedimentos e possíveis resultados dos exames a que foi submetida, avaliando também se possui "Comunicação verbal prejudicada".

A comunicação é outro aspecto de importância ímpar na assistência de enfermagem, sobretudo em relação à parturiente, pois requer do profissional observação minuciosa e competência no mecanismo da comunicação, tanto verbal como não-verbal, já que a parturiente, em geral, está afetada com a situação gerada pelo início do trabalho de parto e as repercussões psicofísicas e sociais que o fato envolve.

- Enfrentamento/Tolerância ao Estresse: a classe Respostas de enfrentamento ao estresse foi selecionada. As crenças culturais sobre o parto podem influenciar a mulher a encará-lo como um acontecimento significativo e ou estressante. Os conceitos sobre o papel da mulher e sua autoconcepção podem afetar seu comportamento durante o trabalho de parto, traduzidos em forma de medo, de ansiedade e comportamentos defensivos. Algumas parturientes recusam ser examinadas, outras pedem ajuda e ainda outras se tornam negativas e infelizes. Além disso, se a mesma vivenciou uma experiência anterior desagradável em relação ao parto, possivelmente terá comportamento negativo durante o trabalho de parto e parto atual. Os dados permitem identificar os possíveis diagnósticos: "Medo", "Ansiedade" e "Enfrentamento individual ineficaz".

- Autopercepção: a classe selecionada foi Auto-estima. Assim, identifica-se o fato da mulher conhecer algo que a amedronta ou ter a percepção de seu próprio valor. Isto pode auxiliar a enfermeira a relacionar tais aspectos a outros dados do comportamento, identificando a possibilidade diagnóstica de "Baixa auto-estima situacional".

Durante a fase ativa do trabalho de parto, a maioria das parturientes manifesta desejo de ter um acompanhamento constante. Hoje, é inquestionável o benefício que a presença de um acompanhante traz à parturiente. En- 
tretanto, não diminui a atenção que a enfermeira obstetra deve proporcionar nesta fase. Pelo contrário, a atenção passa a ser redobrada, pois é importante proporcionar atenção ao acompanhante que, muitas vezes, está emocionalmente mais inseguro que a própria parturiente.

- Princípios de Vida: na Classe Congruência entre valores/crenças/ações, foi introduzido o diagnóstico "Risco de não aderência”. Considerou-se como fator de risco a limitação ao tratamento em decorrência de crença religiosa. Distúrbios hemorrágicos, observáveis na prática obstétrica, que têm como uma das terapêuticas a adoção de transfusão sangüínea, pode ser limitada em decorrência da crença religiosa. Dentro dos princípios que norteiam a bioética, a parturiente tem autonomia para poder recusar receber transfusão de sangue, que é singularidade dos que seguem a religião Testemunhas de Jeová.

- Outros dados: este item no instrumento de levantamento de dados é destinado para outros possíveis diagnósticos identificados.

\section{CONSIDERAÇÕES FINAIS}

O instrumento de coleta de dados apresentado foi oriundo da tese de doutorado apresentada na UNIFESP, o qual mostrou ser pertinente e exequiível nas diferentes fases do trabalho de parto. A elaboração do instrumento com base na estrutura da Taxonomia II-NANDA - Padrões de Saúde Multiaxial permitiu inserir questões que contemplassem aspectos específicos da área obstétrica, exceto no Domínio eliminação, pois houve necessidade de inserir a Classe Sistema Reprodutivo. Facilitou o raciocínio do processo diagnóstico, permitindo sua identificação com maior facilidade, segurança e menor tempo.

\section{REFERÊNCIAS}

1. Stanton M, Paul C, Reeves JS. Um resumo do processo de enfermagem. In: George JB. Teorias de enfermagem: os fundamentos para a prática profissional. Porto Alegre: Arte Médicas; 1993. p. 24.

2. Almeida MA, Soares MAM. Como eu faço o diagnóstico de enfermagem. In: Anais do $4^{\circ}$ Simpósio Nacional sobre Diagnóstico de Enfermagem; 1998; Curitiba. Curitiba: ABEn - Seção PR; 1998. p. 1-7.

3. Marin HF. Documentação de enfermagem: representação da terminologia em um sistema de classificação [tese]. São Paulo: Faculdade de Medicina, Universidade de São Paulo; 2001.

4. Horta WA. O processo de enfermagem. São Paulo: EPU; 1979.

5. Carpenito LJ. Diagnósticos de enfermagem: aplicação à prática clínica. 6a ed. Porto Alegre: Artes Médicas; 1997. Tipos e componentes dos diagnósticos de enfermagem; p. 34-43.

6. Waldow VR. Processo de enfermagem: teoria e prática. Rev Gaúcha Enferm. 1988;9(1):14-22.
7. Iyer PW, Taptich BJ, Bernocchi-Losey D. Processo e diagnóstico de enfermagem. Trad. de Regina Machado Garcez. Porto Alegre: Artes Médicas; 1993. O processo de enfermagem; p.10-5.

8. Azevedo CM. Avaliação da informação registrada em prontuário de pacientes internadas em enfermaria obstétrica de um Hospital-Escola do Município de São Paulo. [Apresentado ao Centro Nacional de Pesquisa Científica-PIBIC. São Paulo: UNIFESP/EPM; 2000].

9. Petrie RH, Williams AM. Trabalho de parto. In: Knuppel RA, Drukker JE. Alto risco em obstetrícia: um enfoque multidisciplinar. 2a ed. Porto Alegre: Artes Médicas; 1995. p. 236-48.

10. North American Nursing Association. Diagnóstico de enfermagem da NANDA: definições classificação: 2001-2002. Trad. de Jeanne Liliane Marlene Michel. Porto Alegre: Artmed; 2002. p. 236-63.

11. Ramos Junior J. Semiotécnica da observação clínica: fisiopatologia dos sintomas e sinais. 7a ed. São Paulo: Sarvier; 1986. Identificação, queixas e duração: história pregressa da moléstia atual; p. 7.

12. Neme B. Obstetrícia básica. São Paulo: Sarvier; 1994. Parto: assistência; p.139.

13. Zamith R, Nazário ACP, Baract EC, Nicolau SM. Corrimento genital. In: Prado FC do, Borges DR, Rothschild HA. Atualização terapêutica: manual prático de diagnóstico e tratamento. São Paulo: Artes Médicas; 2001. p.541-3.

14. Rodrigues RC, Braga AFA. Fisiologia e farmacologia da placenta: efeitos da anestesia sobre útero, placenta e feto. In: Yamashita AM, Gozzani JL. Anestesia em obstetrícia. São Paulo: Atheneu; 1997. p. 13-32.

15. Teixeira MJ, Pimenta CAM. Avaliação do doente com dor. In: Teixeira MJ, Figueiró JAB. Dor. São Paulo: Moreira Jr; 2001. p. 1-11.

16. Burroughs A. Uma introdução à enfermagem materna. 6a ed. Porto Alegre: Artes Médicas; 1995. Assistência de enfermagem durante o trabalho de parto e parto; p. 183-205.

17. Burroughs A. Processo do trabalho de parto normal e parto. In: Burroughs A. Uma introdução à enfermagem materna. 6.ed. Porto Alegre: Artes Médicas; 1995. p.154-69.

18. Gutsche BB. Alterações fisiológicas maternas durante a gravidez. In: Shnider SM, Levnison G. Anestesia em obstetrícia. São Paulo: Manole; 1981. p. 3-11.

19. La Fuente P. Deambulação durante o trabalho de parto e tipos de puxos-sua influência sobre a evolução do parto e o bemestar fetal. In: Sabatino H, Dunn PM, Caldeyro-Barcia R. Parto humanizado: formas alternativas. 2a ed. Campinas: Ed. UNICAMP; 2000. p. 57-80.

20. Gallo M. Efeitos da postura materna durante o trabalho de parto no equilíbrio ácido-base e função pulmonar da mãe. In: Sabatino H, Dunn PM, Caldeyro-Barcia R. Parto humanizado: formas alternativas. Campinas: Ed. UNICAMP; 2000. p. 91-101. 